\title{
Investigation of changes in fractal dimension from layered retinal structures of healthy and diabetic eyes with optical coherence tomography
}

Wei Gao, Valery Zakharov, Oleg Myakinin, Ivan Bratchenko, Dmitry Artemyev, et al.

Wei Gao, Valery P. Zakharov, Oleg O. Myakinin, Ivan A. Bratchenko, Dmitry N. Artemyev, Dmitry V. Kornilin, "Investigation of changes in fractal dimension from layered retinal structures of healthy and diabetic eyes with optical coherence tomography," Proc. SPIE 9541, Optical Coherence Imaging Techniques and Imaging in Scattering Media, 95411I (9 July 2015); doi: $10.1117 / 12.2185222$ 


\section{Investigation of changes in fractal dimension from layered retinal structures of healthy and diabetic eyes with optical coherence tomography" (Withdrawal Notice)}

Proc. SPIE 9541, 95411I (2015)

Online Publication Date: 9 July 2015

Withdrawn from Publication: 18 August 2015

Conference Date: 21-24 June 2015

Conference Location: Munich, Germany

Conference Title: Optical Coherence Imaging Techniques and Imaging in Scattering Media

Conference Chairs: Brett E. Bouma, Maciej Wojtkowski

Wei Gao, Valery Zakharov, Oleg Myakinin, Ivan Bratchenko, Dmitry Artemyev and Dmitry Kornilin Samara State Aerospace Univ. (Russian Federation)

This paper has been withdrawn by the publisher at the request of the authors. 